\title{
APLICAÇÃo DA METODOLOGIA DE PLANEJAMENTO FATORIAL E ANÁLISE DE SUPERFÍCIES DE RESPOSTA PARA OTIMIZAÇÃO DA FERMENTAÇÃO ALCOÓLICA
}

José Alexsandro Silva*

Departamento de Farmácia, Universidade Estadual da Paraíba, Rua Juvêncio Arruda, s/n, Campus Universitário, 58109-790

Campina Grande - PB, Brasil

Bolívar Ponciano Goulart de Lima Damasceno

Centro de Ciências Biológicas e Sociais Aplicadas, Universidade Estadual da Paraíba, João Pessoa-PB, Brasil

Flávio Luiz Honorato da Silva

Unidade Acadêmica de Engenharia Química, Centro de Ciência e Tecnologia, Universidade Federal de Campina Grande, Campina Grande - PB, Brasil

Marta Suely Madruga

Departamento de Tecnologia Química e de Alimentos, Centro de Tecnologia, Campus I, Universidade Federal da Paraíba, João Pessoa - PB, Brasil

Davi Pereira de Santana

Departamento de Ciências Farmacêuticas, Universidade Federal de Pernambuco, Recife-PE, Brasil

Recebido em 14/4/07; aceito em 30/11/07; publicado na web em 24/7/08

\begin{abstract}
APPLICATION OF THE FACTORIAL DESIGN AND RESPONSE SURFACE METHODOLOGY FOR OPTIMIZATION OF ALCOHOLIC FERMENTATION. In the present work, the influence of the amount of nitrogen and phosphorus and degrees Brix on the yield and productivity of alcoholic fermentation has been evaluated. The methodology used was factorial design and response surface analysis. Within the range studied only for phosphorus a statistically significant effect was observed. The broth of sugar cane of the CB 453 variety already possessed enough nitrogen for the fermentation. The mathematical and empirical model was validated for productivity and not for yield. The concentration of alcohol produced in the fermentation was not enough to cause cellular growth inhibition.
\end{abstract}

Keywords: factorial design; productivity; ethanol.

\section{INTRODUÇÃO}

O sistema agroindustrial da cana-de-açúcar já foi tema de inúmeros, profundos e excelentes estudos, realizados quer pela iniciativa privada como pelas Universidades e outras instituições de pesquisa. Além dos aspectos sócio-econômicos, a cultura tem sua importância reconhecida, também, na área ambiental, tendo o álcool etílico como uma fonte renovável de energia menos poluente, a qual já integra a matriz energética nacional e está sendo um dos produtos mais importantes do mercado internacional. ${ }^{1,2}$

A utilização do álcool combustível automotivo no Brasil ganhou importância na década de 70 com o lançamento do Programa do Álcool (Proálcool), criado pelo decreto 76.593/75, cuja finalidade estratégica foi substituir parte da gasolina consumida no mercado interno, reduzindo, assim, as importações de petróleo. ${ }^{3}$

O Brasil posiciona-se no cenário internacional, atualmente, como o maior produtor e exportador mundial de açúcar de cana e o maior produtor e consumidor de álcool, ${ }^{3,4}$ sendo também o único país a introduzir, em larga escala, o álcool como combustível alternativo ao petróleo. ${ }^{1}$ Os países industrializados, como os Estados Unidos da América (EUA), estão interessados na ampliação da produção de álcool etílico do Brasil. A produção de álcool no Brasil, que é da ordem de 17 bilhões de L por safra ${ }^{5,6}$ poderá chegar, em 2010, a uma produção em torno de 30 bilhões de L por safra, ${ }^{3}$ produzida por inúmeras destilarias espalhadas no país. O álcool hidratado é competitivo com a gasolina devido às reduções dos custos no setor (aumento

*e-mail: alexuepb@yahoo.com.br de rendimento e produtividade nas áreas agrícola e industrial), ao aumento do preço do barril de petróleo e às diferenças de tributação da gasolina e do álcool. ${ }^{3}$ Assim, é de vital importância a continuação de estudos na área, buscando aumentar a eficiência do processo.

A produção de álcool no Brasil se faz quase que exclusivamente por fermentação de mosto, constituído por caldo de cana ou melaço, ou ainda por misturas destes dois componentes. Alguns macronutrientes e/ou micronutrientes também são adicionados ao mosto, a fim de complementar as deficiências do meio, quanto a certos elementos indispensáveis às leveduras para uma máxima transformação dos açúcares. O microrganismo agente da fermentação mais utilizado é um fungo leveduriforme, Saccharomyces cerevisiae. ${ }^{1}$

A utilização do método de planejamento fatorial, em estudos que abrangem muitas variáveis, como é o caso da fermentação alcoólica, é importante, pois torna possível o planejamento e a realização de maneira organizada de uma quantidade mínima necessária de experimentos, economizando tempo e recursos financeiros. ${ }^{1,7}$

Diante disso, este estudo teve o objetivo de avaliar a influência das variáveis nitrogênio, fósforo e ${ }^{\circ}$ Brix no rendimento e produtividade e, através da metodologia de planejamento fatorial e análise de superfícies de resposta, propor um modelo probabilístico estatisticamente significativo na fermentação alcoólica em batelada.

\section{PARTE EXPERIMENTAL}

Caldo de cana (substrato)

Foi utilizado como substrato caldo de cana da variedade $\mathrm{CB}$ 
453 do talhão 2045, que é irrigada com vinhaça e rotineiramente utilizado na Destilaria Japungu, Santa Rita - PB, para a produção de álcool.

\section{${ }^{\circ}$ Brix do caldo in natura e do mosto}

Para o doseamento do ${ }^{\circ}$ Brix do caldo in natura, realizado antes das fermentações no laboratório de sacarose da Destilaria Japungu, foi utilizado um refratômetro digital automático da marca ACATEC RDA 8600. O ${ }^{\circ}$ Brix do mosto, dosado durante o processo de fermentação, foi determinado em um sacarímetro de ${ }^{\circ}$ Brix modelo TP 20/C da marca Incoterm.

\section{Açúcares redutores totais (ART)}

A determinação do ART foi feita pelo método titulométrico Lane \& Eynon, no qual o $\mathrm{Cu}^{++}$do reativo de Fehling foi convertido a óxido cuproso $\left(\mathrm{Cu}_{2} \mathrm{O}\right)$ insolúvel, quando aquecido com uma solução de açúcares com grupamento carbonila livre (açúcar redutor). ${ }^{8}$

\section{Nitrogênio total}

O nitrogênio presente nas amostras foi quantificado aplicandose o método de Kjedhal. ${ }^{8}$

\section{Fósforo}

O fósforo foi determinado a partir de uma solução de cinzas do caldo de cana, na qual o fosfato foi transformado em ácido fosfomolibdico e reduzido pelo ácido 1-amino-2-naftol-4-sulfônico a um composto de cor azul, cuja intensidade foi medida espectrofotometricamente a $650 \mathrm{~nm} .^{8}$

\section{Determinação do etanol}

A determinação do etanol foi realizada por cromatografia gasosa, utilizando-se um cromatógrafo GC 90 nas seguintes condições operacionais: temperatura de 130,160 e $86{ }^{\circ} \mathrm{C}$ para injeção, detector e coluna, respectivamente. $\mathrm{O}$ nitrogênio foi utilizado como gás de arraste com uma velocidade de $20 \mathrm{~mL} 40 \mathrm{~s}^{-1}$.

Para a determinação do etanol nas amostras, foi tomada uma alíquota de $23 \mathrm{~mL}$ de vinho delevurado ou centrifugado, juntamente com $2 \mathrm{~mL}$ de acetona PA em um balão volumétrico de $100 \mathrm{~mL}$, completando-se o volume com água destilada. A acetona foi utilizada como padrão interno numa concentração de $8 \%$ v/v e uma solução de etanol $8 \%$ v/v como padrão externo. A partir da percentagem do etanol produzido (\% v/v) e utilizando-se as densidades dos padrões e da água ${ }^{8}\left(\mathrm{~d}_{\text {etanol }}=0,78934 ; \mathrm{d}_{\text {acetona }}=0,792 ; \mathrm{d}_{\text {água }}=1\right)$ foi calculado o peso do etanol em $\mathrm{g} / 100 \mathrm{~g}$ segundo a Equação 1

$$
\mathrm{C}_{\mathrm{g} / 100 \mathrm{~g}}=\frac{\mathrm{C}_{\% \mathrm{v} / \mathrm{v}} \cdot \mathrm{d}_{\text {composto }}}{\mathrm{d}_{\text {solução }}}
$$

onde $\mathrm{d}_{\text {composto }}=$ densidade do etanol; $\mathrm{d}_{\text {solução }}=($ densidade do etanol $\mathrm{x}$ $\%$ volume etanol $)+($ densidade acetona $\mathrm{x} \%$ volume acetona $)+$ (densidade da água x \% volume água).

\section{Cálculo do rendimento}

O rendimento das fermentações foi calculado, utilizando-se os valores de etanol produzido (g/100g) e dos ART no caldo (g/100g), pela Equação 2
Rendimento $\quad(\%)=\frac{\text { Etanol }_{\mathrm{g} / 100 \mathrm{~g}}}{\mathrm{ART}_{\text {caldo }} \cdot 0,5111_{\mathrm{g} / \mathrm{g}}} \times 100$

\section{Cálculo da produtividade}

A produtividade das fermentações foi calculada, utilizando-se os valores de etanol produzido em $(\mathrm{g} / 100 \mathrm{~g})$ e o tempo total de fermentação (h), segundo a Equação 3

Produtividade $=\frac{\text { Etanol }_{\mathrm{g} \mathrm{L}}{ }^{-1}}{\text { Tempo }(\mathrm{h})}$

\section{Delineamento experimental}

O caldo de cana utilizado nas fermentações foi diluído na concentração do ${ }^{\circ}$ Brix desejado e os nutrientes, nitrogênio e fósforo foram adicionados, após o seu doseamento no caldo in natura, conforme o nível do planejamento experimental. Após o ajuste das concentrações destas variáveis de entrada, os caldos foram esterilizados em autoclave a $121{ }^{\circ} \mathrm{C}, 1 \mathrm{~atm}$ de pressão por $30 \mathrm{~min}$, sendo posteriormente resfriados à temperatura ambiente.

\section{Processo de fermentação}

Para o processo de fermentação foi utilizada a cepa Sacharomyces cerevisiae IA 1234, obtida a partir do fermento (leite puro) proveniente da fermentação alcoólica da Destilaria Japungu. Para cada fermentação foram adicionados $250 \mathrm{~mL}$ de leite puro (fermento), que corresponde a uma concentração de $10 \%$ v/v do caldo.

As fermentações foram realizadas em balão de fundo chato de 6000 mL, tampado com algodão hidrófilo. O acompanhamento do processo de fermentação foi realizado por medição horária do ${ }^{\circ}$ Brix, considerando-se como o fim da fermentação quando o consumo de açúcares cessava ou quando o valor do ${ }^{\circ}$ Brix repetia por no mínimo duas vezes. ${ }^{1,9}$

\section{RESULTADOS E DISCUSSÃO}

\section{Produção de etanol}

Durante o processo da fermentação alcoólica origina-se uma série de compostos que podem atuar como inibidores potenciais, entre eles podem ser citados os metabólitos secundários contaminantes totais e, até mesmo, o etanol produzido no processo. ${ }^{1}$ A natureza e concentração destes compostos dependem das condições do processo, como temperatura do meio e tempo de fermentação. ${ }^{10}$

Os valores das concentrações de etanol expressos em g/100 g resultantes das 16 fermentações realizadas no presente trabalho estão descritos na Tabela 1. O teor alcoólico médio foi de 6,02 \pm $1,19 \mathrm{~g} / 100 \mathrm{~g}$, o que corresponde a um valor corrigido de densidade a cerca de 7,5\% v/v. As fermentações desenvolveram-se dentro dos parâmetros normais e com teor alcoólico de 6 a $9 \%$ v/v, com substrato variando de 10 a $20 \% \mathrm{v} / \mathrm{v}$, relatados na literatura. ${ }^{11} \mathrm{O}$ etanol é o metabólito produzido em maior quantidade em uma fermentação alcoólica, podendo ser tóxico em uma determinada concentração, causando redução na viabilidade celular das leveduras. ${ }^{12}$

$\mathrm{Na}$ Tabela 1 verifica-se que os teores alcoólicos obtidos, em torno de 7,5\% v/v, não foram suficientes para causar grande inibição da levedura durante as fermentações. Teor alcoólico de 11,1\% pode causar inibição no crescimento celular e quando atinge valo- 
Tabela 1. Matriz do planejamento fatorial com a produção de etanol em g/100 g

\begin{tabular}{lccc}
\hline Nitrogênio & Fósforo & ${ }^{\circ}$ Brix & Etanol $(\mathrm{g} / 100 \mathrm{~g} \pm \mathrm{DP})^{*}$ \\
\hline-1 & -1 & -1 & $4,52 \pm 0,04$ \\
+1 & -1 & -1 & $4,64 \pm 0,14$ \\
-1 & +1 & -1 & $5,01 \pm 0,06$ \\
+1 & +1 & -1 & $4,99 \pm 0,02$ \\
-1 & -1 & +1 & $7,14 \pm 0,09$ \\
+1 & -1 & +1 & $7,09 \pm 0,13$ \\
-1 & +1 & +1 & $7,22 \pm 0,11$ \\
+1 & +1 & +1 & $7,33 \pm 0,01$ \\
$-1,682$ & 0 & 0 & $6,23 \pm 0,03$ \\
$+1,682$ & 0 & 0 & $6,11 \pm 0,01$ \\
0 & $-1,682$ & 0 & $6,01 \pm 0,07$ \\
0 & $+1,682$ & 0 & $6,19 \pm 0,05$ \\
0 & 0 & $-1,682$ & $4,02 \pm 0,04$ \\
0 & 0 & $+1,682$ & $8,31 \pm 0,02$ \\
0 & 0 & 0 & $5,76 \pm 0,03$ \\
0 & 0 & 0 & $5,79 \pm 0,001$ \\
\hline Valor médio $(\mathrm{X})$ & - & - & $6,02 \pm 1,19$ \\
\hline
\end{tabular}

* Os valores de etanol (g/100 g) são referentes à média de dois cromatogramas

res acima de 14,5\% não ocorre mais produção de etanol. ${ }^{13}$

As leveduras não se reproduzem quando a concentração do etanol ultrapassa valores de $13,7 \%$ v/v. ${ }^{14}$ As enzimas glicolíticas das mesmas são inibidas em concentrações de etanol em torno de $12 \% \mathrm{v} / \mathrm{v} .{ }^{15}$ Teor alcoólico de $12 \% \mathrm{v} / \mathrm{v}$ é considerado normal em fermentações alcoólicas. ${ }^{16} \mathrm{O}$ etanol é mais tóxico quando gerado endogenamente, ${ }^{17,18}$ pois seu efeito tóxico estar relacionado à interação com determinadas zonas hidrofóbicas das células, causando perda da integridade da membrana, afetando sua capacidade de agir como uma barreira seletiva. ${ }^{19}$ Concentrações altas de açúcares redutores no meio aumentam a produção de etanol e de outros componentes que levam à inibição do crescimento celular. ${ }^{20}$

Em estudo realizado sobre a determinação da toxicidade do etanol, através de cinética de inativação do Saccharomyces cerevisiae, foi observado que estes microrganismos resistem a uma concentração de 22 a $25 \%$ de etanol, desde que o meio possua oxigênio (aeração) e certa quantidade de lipídios. ${ }^{21}$ De forma semelhante, existem dados na literatura ${ }^{19}$ que demonstram a capacidade de determinados microrganismos, entre eles os do gênero Saccharomyces, metabolizarem, a partir de sistemas enzimáticos próprios, certos alcoóis secundários. Deste modo, pode-se presumir que não houve inibição do crescimento celular neste estudo.

\section{Planejamento experimental}

Para avaliar a influência das variáveis independentes - ${ }^{\circ}$ Brix, nitrogênio e fósforo - sobre as variáveis dependentes rendimento e produtividade, foi elaborado um planejamento fatorial de 2 níveis $(-1$ e +1$)$ mais configuração estrela, aqui chamado de modelo de $2^{\mathrm{a}}$ ordem, totalizando 16 experimentos laboratoriais, executados aleatoriamente e em duplicatas, conforme matriz do planejamento fatorial mostrado na Tabela 2.

$\mathrm{Na}$ Tabela 3 estão expressos os valores reais e os níveis dos fatores estudados nos 16 experimentos. As faixas estudadas foram definidas tendo como base valores comumente utilizados em muitas destilarias de álcool, principalmente da região Nordeste e, em especial, na Destilaria Japungu.

Os modelos matemáticos empíricos, codificados e de $2^{\mathrm{a}}$ or-
Tabela 2. Matriz do planejamento fatorial $2^{3}+$ configuração estrela com as respostas rendimento e produtividade

\begin{tabular}{lcccr}
\hline Nitrogênio & Fósforo & 'Brix & $\begin{array}{r}\text { Rendimento } \\
(\%)^{*}\end{array}$ & $\begin{array}{r}\text { Produtividade } \\
\left(\mathrm{g} \mathrm{L}^{-1} \mathrm{~h}^{-1}\right)\end{array}$ \\
\hline-1 & -1 & -1 & 90,24 & 5,09 \\
+1 & -1 & -1 & 91,95 & 5,19 \\
-1 & +1 & -1 & 94,14 & 6,21 \\
+1 & +1 & -1 & 94,45 & 6,23 \\
-1 & -1 & +1 & 90,16 & 6,25 \\
+1 & -1 & +1 & 89,55 & 5,06 \\
-1 & +1 & +1 & 91,09 & 6,19 \\
+1 & +1 & +1 & 91,88 & 5,86 \\
$-1,682$ & 0 & 0 & 93,84 & 5,31 \\
$+1,682$ & 0 & 0 & 92,64 & 5,09 \\
0 & $-1,682$ & 0 & 90,37 & 5,64 \\
0 & $+1,682$ & 0 & 93,73 & 6,63 \\
0 & 0 & $-1,682$ & 91,31 & 5,13 \\
0 & 0 & $+1,682$ & 93,74 & 5,60 \\
0 & 0 & 0 & 86,64 & 6,39 \\
0 & 0 & 0 & 87,16 & 6,43 \\
\hline
\end{tabular}

* Os valores de Rendimento e Produtividade são referentes à média de dois cromatogramas

Tabela 3. Valores reais e níveis dos fatores estudados para o planejamento fatorial completo $\left(2^{3}\right)+$ configuração estrela do processo fermentativo

\begin{tabular}{lccccc}
\hline Variáveis & $\begin{array}{c}\text { Nível } \\
-1,682\end{array}$ & Nível -1 & $\begin{array}{c}\text { Ponto } \\
\text { central }\end{array}$ & Nível +1 & $\begin{array}{c}\text { Nível } \\
+1,682\end{array}$ \\
\hline $\mathrm{N}\left(\mathrm{g} \mathrm{L}^{-1}\right)$ & 0,1 & 0,3 & 0,6 & 0,9 & 1,1 \\
$\mathrm{P}\left(\mathrm{g} \mathrm{L}^{-1}\right)$ & 0,002 & 0,05 & 0,12 & 0,19 & 0,24 \\
${ }^{\circ}$ Brix $(\%)$ & 10 & 12 & 15 & 18 & 20 \\
\hline
\end{tabular}

dem, encontrados, com os seus respectivos coeficientes estatísticos estão apresentados nas Equações 4 e 5, onde os valores em negrito são os parâmetros estatisticamente significativos. Estes modelos foram obtidos a partir das regressões não lineares (modelo quadrático) dos dados experimentais, utilizando o software Statistica versão 5.0.

Rend $=(\mathbf{8 7 , 0 2} \pm \mathbf{0 , 8 3})-(0,74 \mathrm{~N} \pm 0,32)+(\mathbf{1 , 1 2 P} \pm \mathbf{0 , 3 2})+(0,46$

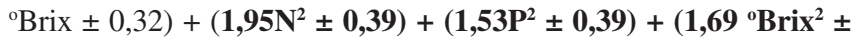
0,39) $-(0,39$ N.P $\pm 0,42)-\left(0,23 N\right.$. ${ }^{\circ}$ Brix $\left.\pm 0,42\right)-\left(0,00 P .{ }^{\circ}\right.$ Brix \pm $0,42)$

Prod $=(\mathbf{6 , 3 9} \pm \mathbf{0 , 2 4})+(0,019 \mathrm{~N} \pm 0,09)+(\mathbf{0 , 3 3 P} \pm \mathbf{0 , 0 9})-(0,04$ ${ }^{\circ}$ Brix $\left.\pm 0,09\right)-\left(\mathbf{0 , 3 7 N ^ { 2 }} \pm \mathbf{0 , 1 1}\right)-\left(0,04 \mathrm{P}^{2} \pm 0,11\right)-\left(\mathbf{0 , 3 1}{ }^{\circ} \mathbf{B r i x}^{2} \pm\right.$ 0,11) $-(0,18 \mathrm{~N} . \mathrm{P} \pm 0,12)-\left(0,20 \mathrm{~N} .{ }^{\circ}\right.$ Brix $\left.\pm 0,12\right)+\left(0,10\right.$ P. ${ }^{\circ}$ Brix \pm $0,12)$

Os resultados da análise de variância (ANOVA) para a resposta rendimento estão descritos na Tabela 4 , onde se observa que o coeficiente de correlação e a percentagem de variância explicada não foram satisfatórios, explicando apenas $78,91 \%$ de variância com um coeficiente de correlação de 0,89 . Observa-se, também, que o valor de $\mathrm{F}_{\text {calculado }}=2,49$ é menor que o valor de $\mathrm{F}_{\text {Tabelado }}=4,1 \mathrm{a}$ um nível de confiança de $95 \%$. Deste modo, pode-se afirmar que o referido modelo não apresentou significância estatística e que o mesmo não pode ser utilizado para fins preditivos.

Na Tabela 5, que apresenta os resultados da Análise de Variância (ANOVA) para a variável produtividade, verifica-se que o modelo 
Tabela 4. Análise de Variância (ANOVA) para a variável resposta rendimento

\begin{tabular}{|c|c|c|c|c|c|}
\hline & $\begin{array}{c}\text { Soma } \\
\text { quadrática }\end{array}$ & $\begin{array}{l}\text { Grau de } \\
\text { liberdade }\end{array}$ & $\begin{array}{c}\text { Média } \\
\text { quadrática }\end{array}$ & Teste F & $\mathrm{p}$ \\
\hline N (linear) & 7,496 & 1 & 7,496 & 5,31 & 0,06 \\
\hline N (quadrático) & 35,08 & 1 & 35,08 & 24,86 & 0,002 \\
\hline P (linear) & 17,16 & 1 & 17,16 & 12,16 & 0,01 \\
\hline P (quadrático) & 21,55 & 1 & 21,55 & 15,27 & 0,007 \\
\hline${ }^{\circ}$ Brix (linear) & 2,89 & 1 & 2,89 & 2,05 & 0,202 \\
\hline${ }^{\circ}$ Brix (quadrático) & o) 26,56 & 1 & 26,56 & 18,82 & 0,005 \\
\hline $\mathrm{N} \times \mathrm{P}$ & 1,23 & 1 & 1,23 & 0,87 & 0,39 \\
\hline $\mathrm{N} x{ }^{\circ}$ Brix & 0,42 & 1 & 0,42 & 0,29 & 0,603 \\
\hline $\mathrm{P} x{ }^{\circ}$ Brix & 0,00 & 1 & 0,00 & 0,00 & 1,00 \\
\hline Resíduo & 8,47 & 6 & 1,41 & - & - \\
\hline Total & 83,83 & 15 & - & - & - \\
\hline \multicolumn{2}{|c|}{ \% variância explicada } & \multicolumn{2}{|c|}{ Coeficiente correlação } & $\mathrm{F}_{\text {calculado }}$ & $\begin{array}{l}\mathrm{F}_{\text {Tabelado }} \\
95 \%\end{array}$ \\
\hline \multicolumn{2}{|l|}{78,91} & \multicolumn{2}{|c|}{0,89} & 2,49 & 4,10 \\
\hline
\end{tabular}

Tabela 5. Análise de Variância (ANOVA) para a variável resposta produtividade

\begin{tabular}{|c|c|c|c|c|c|}
\hline & $\begin{array}{c}\text { Soma } \\
\text { quadrática }\end{array}$ & $\begin{array}{l}\text { Grau de } \\
\text { liberdade }\end{array}$ & $\begin{array}{c}\text { Média } \\
\text { quadrática }\end{array}$ & Teste F & $\mathrm{p}$ \\
\hline $\mathrm{N}$ (linear) & 0,01 & 1 & 0,01 & 0,04 & 0,84 \\
\hline N (quadrático) & 1,27 & 1 & 1,27 & 10,69 & 0,02 \\
\hline $\mathrm{P}$ (linear) & 1,52 & 1 & 1,52 & 12,80 & 0,01 \\
\hline $\mathrm{P}$ (quadrático) & 0,01 & 1 & 0,01 & 0,13 & 0,73 \\
\hline${ }^{\circ}$ Brix (linear) & 0,03 & 1 & 0,03 & 0,23 & 0,65 \\
\hline${ }^{\circ}$ Brix (quadrático) & 0,90 & 1 & 0,90 & 7,59 & 0,03 \\
\hline $\mathrm{N} \times \mathrm{P}$ & 0,25 & 1 & 0,25 & 2,11 & 0,19 \\
\hline $\mathrm{N} x{ }^{\circ}$ Brix & 0,34 & 1 & 0,34 & 2,82 & 0,14 \\
\hline $\mathrm{P} \times{ }^{\circ}$ Brix & 0,08 & 1 & 0,08 & 0,63 & 0,45 \\
\hline Resíduo & 0,71 & 6 & 0,12 & - & - \\
\hline Total & 4,81 & 15 & - & - & - \\
\hline \multicolumn{2}{|c|}{ \% variância explicada } & \multicolumn{3}{|c|}{ Coeficiente correlação $\mathrm{F}_{\text {calculado }}$} & $\begin{array}{l}\mathrm{F}_{\text {Tabelado }} \\
95 \%\end{array}$ \\
\hline \multicolumn{2}{|l|}{92,29} & \multicolumn{2}{|c|}{0,96} & 7,99 & 4,10 \\
\hline
\end{tabular}

pode ser usado para fins preditivos, uma vez que o mesmo apresentou um coeficiente de correlação de 0,96 com uma percentagem de variância explicada de 92,29. O teste $\mathrm{F}$ também mostra que o valor de $\mathrm{F}_{\text {calculado }}=7,99$ é maior que o valor de $\mathrm{F}_{\text {thelado }}=4,1$, aproximadamente 2 vezes, com $95 \%$ de confiança, mostrando que o modelo foi bem ajustado para esta variável, sendo este estatisticamente significativo.

Considerando que o modelo quadrático foi validado para a variável produtividade, o mesmo foi usado para gerar as superfícies de resposta, tendo o objetivo de otimizar (maximizar) o processo. ${ }^{1}$ As superfícies de resposta para variável rendimento não foram construídas, pois o modelo não apresentou significância estatística, como discutido acima.

A Figura 1 apresenta os efeitos do ${ }^{\circ}$ Brix e fonte de fósforo na produtividade, estando o nitrogênio fixado em $0,6 \mathrm{~g} \mathrm{~L}^{-1}$ (ponto central). Observa-se que se fixando a concentração de fósforo acima de $0,12 \mathrm{~g} \mathrm{~L}^{-1}$, para qualquer valor de ${ }^{\circ} \mathrm{Brix}$, obtém-se produtividade maximizada de aproximadamente $6,6 \mathrm{~g} \mathrm{~L}^{-1} \mathrm{~h}^{-1}$.

A Figura 2 apresenta os efeitos da fonte nitrogênio e do ${ }^{\circ}$ Brix na produtividade tendo o valor de fósforo fixado no ponto central

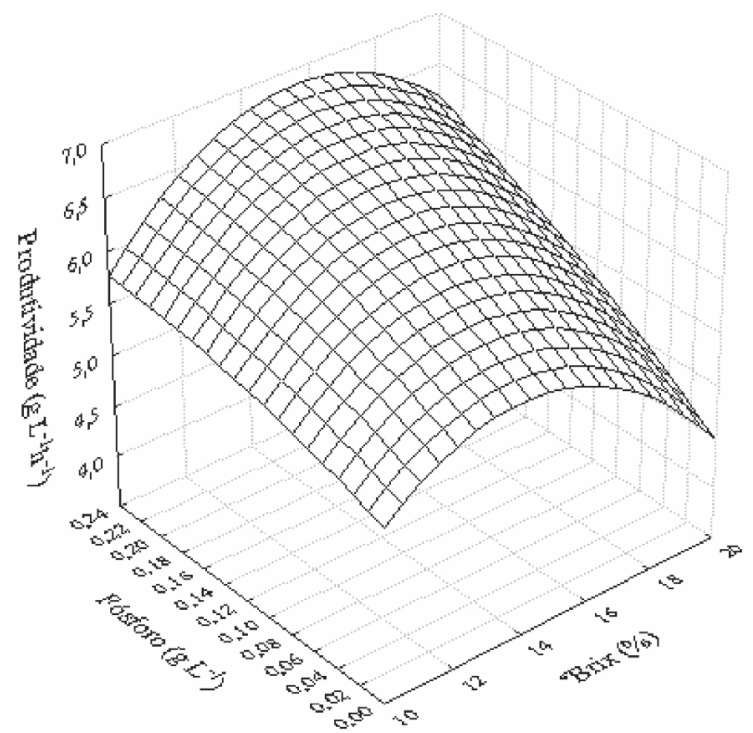

Figura 1. Efeitos do ${ }^{\circ}$ Brix e do fósforo na produtividade $\left(g L^{-1} h^{-1}\right)$ para o nitrogênio fixado no ponto central

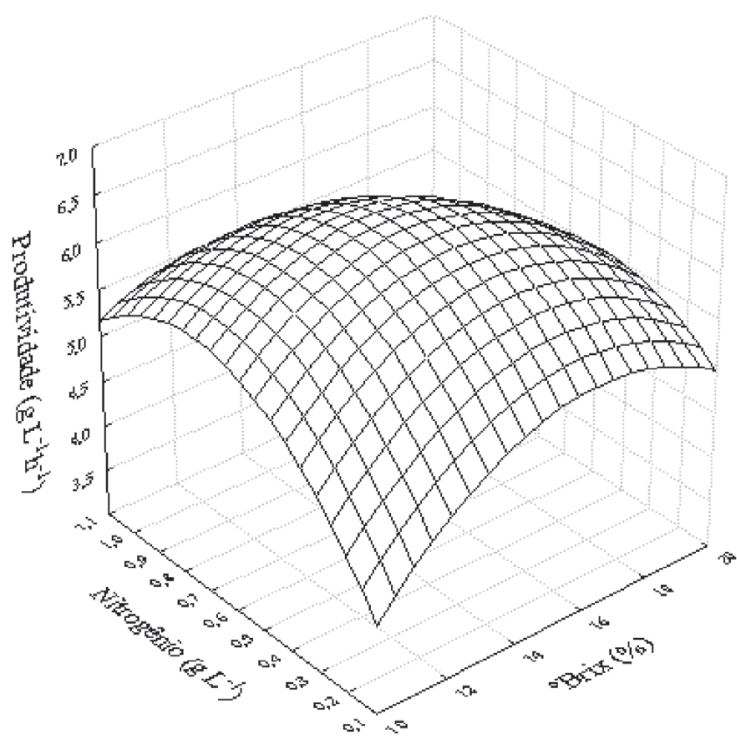

Figura 2. Efeitos do ${ }^{\circ}$ Brix e do nitrogênio na produtividade $\left(g L^{-1} h^{-1}\right)$ para o fósforo fixado no ponto central

$\left(0,12 \mathrm{~g} \mathrm{~L}^{-1}\right)$. Produtividade em torno de $6,2 \mathrm{~g} \mathrm{~L}^{-1} \mathrm{~h}^{-1}$ é obtida quando $\mathrm{o}{ }^{\circ}$ Brix se encontra em $15 \%$ e o nitrogênio em $0,6 \mathrm{~g} \mathrm{~L}^{-1}$, ou seja, os valores das variáveis fixadas no ponto central.

A Figura 3 apresenta os efeitos das fontes de fósforo e nitrogênio sobre a resposta produtividade. Observa-se que se fixando o valor de fósforo acima de $0,19 \mathrm{~g} \mathrm{~L}^{-1}$ e nitrogênio em $0,6 \mathrm{~g} \mathrm{~L}^{-1}$, obtém-se valor máximo de produtividade de $6,6 \mathrm{~g} \mathrm{~L}^{-1} \mathrm{~h}^{-1}$.

Para obter maior eficiência (produtividade e rendimento) no processo em estudo, deve-se operar com concentração de ${ }^{\circ}$ Brix em torno de $15 \%$, fontes de fósforo e nitrogenio de 0,19 e $0,6 \mathrm{~g} \mathrm{~L}^{-1}$, respectivamente, conseguindo otimizar a produtividade em $6,6 \mathrm{~g}$ $\mathrm{L}^{-1} \mathrm{~h}^{-1}$ e rendimento em $88 \%$. O aumento do ${ }^{\circ}$ Brix melhora a produtividade, mesmo que o efeito seja pouco pronunciado. A concentração de açúcares redutores totais no meio é diretamente proporcional à produção de álcool, onde com valores elevados de ${ }^{\circ} \mathrm{Brix}$ obtém-se teores elevados de álcool, tendo como consequiência altos valores de produtividade. ${ }^{12}$ 


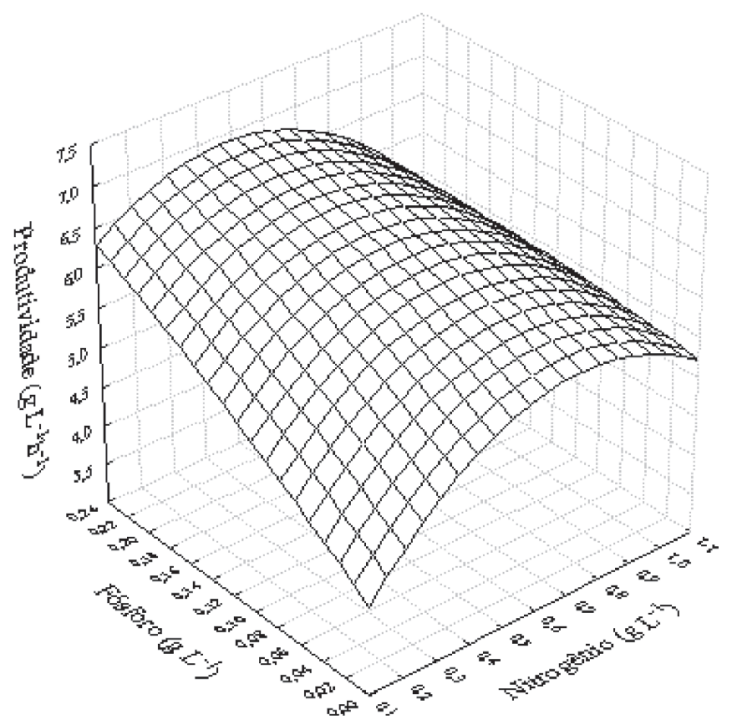

Figura 3. Efeitos do fósforo e nitrogênio na produtividade $\left(g L^{-1} h^{-1}\right)$ para o ${ }^{\circ}$ Brix fixado no ponto central

\section{CONCLUSÃO}

As fontes de nutrientes nitrogênio e fósforo, na resposta rendimento e produtividade, na faixa estudada, só demonstraram efeito significativo de $1^{\underline{a}}$ ordem na variável fósforo, mostrando que o caldo de cana da variedade CB 453 já possui quantidades suficientes do nutriente nitrogênio para que ocorra uma boa fermentação. $\mathrm{O}$ modelo matemático probabilístico e empírico foi validado para a variável resposta produtividade e não para o rendimento e os teores alcoólicos obtidos nas fermentações não foram suficientes para causar inibição celular.

\section{AGRADECIMENTOS}

À Destilaria Japungu - Santa Rita-PB pelo suporte financeiro do Projeto.

\section{REFERÊNCIAS}

1. Silva, J. A.; Silva, F. L. H.; Alves, R. R. N.; Santana, D. P.; Quim. Nova 2006, 29, 695.

2. Fernandes, A. C.; STAB 2003, 2, 237.

3. Souza, R. R.; Dissertação de Mestrado, Universidade Federal do Rio de Janeiro, Brasil, 2006.

4. Saito, J. R.; Dissertação de Mestrado, Universidade Federal de São Carlos, Brasil, 2000.

5. Pereira, E. A.; Tavares, M. F. M.; Stevanato, A.; Cardoso, A. A.; Quim. Nova 2006, 29, 66.

6. Bastos, V. D.; Alcoolquímica 2007, 25, 5.

7. Barros Neto, B.; Scarminio, I. S.; Bruns, R. E.; Como Fazer Experimentos, Ed. da Unicamp: Campinas, 2003

8. Brasil, Ministério da Saúde; Métodos Físico-Químicos para Análises de Alimentos, IV Edição, São Paulo, 2005.

9. Silva, J. A.; Dissertação Mestrado, Universidade Federal da Paraíba, Brasil, 1999.

10. Rossel, C. E. V.; Industrial Perspectives for Bioethanol, Ed. Telma Franco: São Paulo, 2006

11. Castro, H. F.; Tese de Doutorado, University of Manchester, Inglaterra, 1985.

12. Silva, F. L. H.; Tese de Doutorado, Universidade Estadual de Campinas, Brasil, 1998.

13. Ghose, T. K.; Tyagi, R. D.; Biotechnol. Bioeng. 1979, 21, 1387.

14. Luong, J. H. T.; Biotechnol. Bioeng. 1985, 27, 280.

15. Millar, D. G.; Griffiths-Smith, K.; Algar, E.; Scopes, R. K.; Biotechnol. Lett. 1982, 4, 601.

16. Drysdale, G. S.; Fleet, G. H.; J. Bacteriol. 1989, 67, 471.

17. Hoppe, G. K.; Hansford, C. S.; Biotechnol. Lett. 1982, 4, 39.

18. Nagodawithana, T. W.; Steinkraus, K. H.; Appl. Microbiol. Biotechnol. 1990, 34, 375

19. Dominguez, J. M. O.; Tese de Doutorado, Universidad Complutense de Madrid, Espanha, 2003

20. Okolo, B.; Johnston, J. R.; Berry, D. R.; Biotechnol. Lett. 1987, 9, 431.

21. Pina, C.; Santos, C.; Couto, J. A; Hogg, T.; Food Microbiol. 2004, 21, 439. 